\title{
CRIAÇÃO DE UM MANUAL PARA POSICIONAMENTO CIRÚRGICO: RELATO DE EXPERIÊNCIA
}

\author{
Development of a handbook of surgical positioning: experience report \\ Creación de un manual para posicionamiento quirúrgico: relato de experiencia \\ Cristina Silva Sousa ${ }^{1 *}$, Daniela Magalhães Bispo², Andrea Alfaya Acunã ${ }^{3}$
}

RESUMO: Objetivo: Relatar a experiência da criação de um manual de posicionamento cirúrgico. Método: Relato de experiência da construção de um manual de posicionamento cirúrgico para direcionar os profissionais de enfermagem, com base no fundamento teórico e na prática clínica da equipe de enfermagem de um hospital filantrópico de grande porte localizado no município de São Paulo. O guia foi desenvolvido como oportunidade de melhorar a prevenção de lesões por posicionamento cirúrgico. Resultados: O manual é composto de 64 páginas, em apresentação paisagem, colorido e com ilustrações, validado pela gerente e coordenadora do bloco operatório. Contém introdução, avaliação de risco, dispositivos e práticas recomendadas. Para cada posição cirúrgica, é demonstrado como realizar o posicionamento e os riscos envolvidos. Ao final do documento, são informados os dispositivos de prevenção utilizados pela instituição. Conclusão: A criação de manual de posicionamento permite à equipe de enfermagem perioperatória o direcionamento adequado para o posicionamento cirúrgico e, consequentemente, para a prevenção de lesões por pressão decorrentes do posicionamento equivocado. Palavras-chave: Enfermagem perioperatória. Posicionamento do paciente. Lesão por pressão.

ABSTRACT: Objective: To report the experience of developing a handbook of surgical positioning. Method: Experience report on the development of a handbook of surgical positioning to guide nursing professionals, based on theoretical foundation and clinical practice of the nursing staff in a large philanthropic hospital located in the city of São Paulo. We developed the guide as an opportunity to improve prevention of injuries caused by surgical positioning. Results: The handbook comprises 64 pages in landscape orientation, colored, and illustrated, validated by the surgical block manager and coordinator. It includes an introduction, risk assessment, devices, and recommended practices. For each surgical position, it demonstrates how to place the patient and the risks involved. The end of the document brings information about prevention devices used by the institution. Conclusion: The development of a handbook of positioning allows the perioperative nursing team to have proper guidance on surgical positioning and, consequently, prevent pressure ulcers caused by mistaken positioning.

Keywords: Perioperative nursing. Patient positioning. Pressure ulcer.

RESUMEN: Objetivo: Relatar la experiencia de la creación de un manual de posicionamiento quirúrgico. Método: Relato de experiencia de la construcción de un manual de posicionamiento quirúrgico para direccionar los profesionales de enfermería, con base en el fundamento teórico y en la práctica clínica del equipo de enfermería de un hospital filantrópico de grande porte localizado en el municipio de São Paulo. La guía fue desarrollada como oportunidad de mejorar la prevención de lesiones por posicionamiento quirúrgico. Resultados: El manual está compuesto por 64 páginas, en presentación paisaje, colorido y con ilustraciones, validado por la gerente y coordinadora del bloque operatorio. Contienen introducción, evaluación de riesgo, dispositivos y prácticas recomendadas. Para cada posición quirúrgica, es demostrado como realizar el posicionamiento y los riesgos involucrados. Al final del documento, son informados los dispositivos de prevención utilizados por la institución. Conclusión: La creación de manual de posicionamiento permite al equipo de enfermería perioperatoria el direccionamiento adecuado para el posicionamiento quirúrgico y, consecuentemente, para la prevención de lesiones por presión decurrentes del posicionamiento equivocado.

Palabras clave: Enfermería perioperatoria. Posicionamiento del paciente. Úlcera por presión.

\footnotetext{
'Enfermeira; doutora em Ciências da Saúde pela Escola de Enfermagem da Universidade de São Paulo; enfermeira assistencial do centro cirúrgico do Hospital Sírio-Libanês - São Paulo (SP), Brasil. ${ }^{2}$ Enfermeira; mestre em Saúde Coletiva pelo Instituto Sírio-Libanês de Ensino e Pesquisa; coordenadora do Bloco Operatório do Hospital Sírio-Libanês - São Paulo (SP), Brasil.

${ }^{3}$ Enfermeira; gerente do Bloco Operatório do Hospital Sírio-Libanês - São Paulo (SP), Brasil.

*Autor correspondente: crissousa@usp.br

Recebido: 05/11/2017 - Aprovado: 20/06/2018

DOI: $10.5327 / 21414-4425201800030009$
} 


\section{INTRODUÇÃO}

O posicionamento cirúrgico é um procedimento executado pelos profissionais de enfermagem, em conjunto com as equipes anestésica e cirúrgica, durante o período intraoperatório ${ }^{1}$. Devem ser consideradas a anatomia do paciente, suas limitações de movimento e a área de acesso para o cirurgião e seus asistentes ${ }^{2}$.

Os objetivos do posicionamento incluem: providenciar adequada exposição do sítio cirúrgico; manter a dignidade do paciente durante a exposição corporal; proporcionar ventilação e manutenção da via aérea pérvia; prover acesso venoso; monitorizar e controlar as eliminações fisiológicas, de acordo com o posicionamento e fácil acesso para avaliação e mensuração de débito; observar e proteger dedos e genitais; manter a circulação; e proteger músculos, nervos e proeminências ósseas ${ }^{3}$.

As complicações do posicionamento cirúrgico são descritas, principalmente, na forma de lesões por pressão (LP $)^{4}$, mas também podem resultar em dor musculoesquelética, deslocamento de articulações, danos em nervos periféricos, comprometimento cardiovascular e pulmonar e até síndrome compartimental ${ }^{1}$.

O posicionamento cirúrgico adequado garante eficiência e segurança durante o procedimento e é um dos principais indicadores de qualidade do cuidado na assistência perioperatória ${ }^{5}$. Para o apropriado posicionamento cirúrgico, os dispositivos recomendados para auxiliar e prevenir as LP são os posicionadores viscoelásticos, curativos adesivos profiláticos, posicionadores específicos e espumas. Está contraindicado o uso de tecidos ${ }^{2}$.

Visando prever o risco do paciente para desenvolver LP, escalas de avaliação de risco têm sido aplicadas pela enfermagem perioperatória, instrumento cuja função é determinar o risco da predisposição da lesão por posicionamento. Entre as escalas, há a Munro Scale ${ }^{6}$, que consiste em três períodos de avaliação (pré-, intra e pós-operatório), porém ainda não é validada para o português; e a Escala de Avaliação de Risco para o Desenvolvimento de Lesões Decorrentes do Posicionamento Cirúrgico (ELPO) $)^{7}$ que abrange a aplicação intraoperatória e uma somatória de itens de avaliação para estabelecer escore de menor risco e maior risco para desenvolver lesão por posicionamento, baseado na nota de corte de 19 pontos. Assim, é possível definir o escore de risco do paciente para desenvolver LP e alertar o profissional para pacientes com maior risco, a fim de buscar melhores estratégias de prevenção.

Em uma revisão sistemática sobre os eventos adversos (EA) cirúrgicos, os autores descrevem a ocorrência de LP como o mais comum e de possível prevenção ${ }^{8}$. Uma revisão integrativa sobre os cuidados de enfermagem no transoperatório detalha complicações resultantes do posicionamento cirúrgico relacionadas à pele, e seus resultados evidenciaram estudos com incidência de LP grau I ${ }^{9}$.

Lesões por posicionamento cirúrgico são classificadas como eventos com dano ao paciente e requerem medidas preventivas aplicadas pelos enfermeiros perioperatórios. Dessa forma, a idealização de um manual de posicionamento cirúrgico visa direcionar a equipe de enfermagem para realizar corretamente o posicionamento e prevenir a ocorrência de EA cirúrgicos associados à LP.

\section{OBJETIVO}

Relatar a experiência da criação de um manual de posicionamento cirúrgico em um hospital de alta complexidade de São Paulo.

\section{MÉTODO}

Trata-se de um relato de experiência sobre a construção de um manual de posicionamento cirúrgico com base no fundamento teórico e na prática clínica da equipe de enfermagem de um hospital de alta complexidade, filantrópico e de grande porte localizado no município de São Paulo, no período de setembro e outubro de 2017.

Esse manual foi desenvolvido por uma enfermeira perioperatória com mais de dez anos de experiência, como oportunidade de melhoria da prevenção de lesões por posicionamento cirúrgico.

Entre os indicadores de assistência do centro cirúrgico (CC), são registradas as ocorrências com lesões por posicionamento. Apesar de não ser um indicador específico, uma vez que o relato dessa ocorrência é parte do registro de LP, observa-se a presença do EA em risco leve e moderado.

Dessa forma, para minimizar a incidência das LP, foi proposto construir um manual de posicionamento cirúrgico para direcionar a equipe de enfermagem à aplicação de melhores práticas de prevenção de lesões decorrentes do posicionamento.

\section{Fase l: conteúdo do manual de posicionamento}

Para a construção do material, iniciou-se a leitura das recomendações de associações de enfermagem perioperatória americana, europeia e brasileira, além de revisões integrativas e literatura nacional e internacional, com associação à prática clínica executada na instituição. Buscavam-se, com 
isso, recomendações nacionais e internacionais de melhores práticas para prevenção de lesões por posicionamento.

A seguir, foram elencados os pontos gerais para a introdução do material, como avaliação de risco, escala de avaliação, dispositivos para posicionamento e práticas gerais para quaisquer posições cirúrgicas.

Posteriormente, foram identificadas as posições cirúrgicas e a sequência para o posicionamento adequado, bem como os riscos envolvidos. Em reunião com a coordenadora e gerente do bloco cirúrgico (BC), decidiu-se pela inclusão de imagens, na forma de desenhos, para posições cirúrgicas e ilustrações, por meio de fotografias, para os dispositivos de prevenção de lesões, disponíveis na instituição.

\section{Fase II: construção do material}

A ideia de construção do material deveria abranger os diferentes níveis profissionais. Com isso, a linguagem do manual é simples e direcionada em tópicos, de modo a facilitar a identificação dos passos para cada posicionamento cirúrgico. O manual foi realizado em Microsoft Power Point, fonte tamanho 22, em formato de apresentação.

Finalizados o conteúdo e o formato do manual proposto, este passou por validação das enfermeiras coordenadora e gerente do BC, que possuem vasta experiência profissional na enfermagem perioperatória, para análise do conteúdo, formato e leiaute.

Ao término do processo, o material no formato propostoinstituído foi impresso colorido em papel sulfite, formato paisagem e encadernado em espiral. Assim, foi apresentado à equipe de enfermagem para leitura e disponibilizado como material de consulta no posto de enfermagem do CC para acesso diário por qualquer profissional.

\section{RESULTADOS}

O manual de posicionamento é composto de 64 páginas, sendo cada tópico constituído de uma cor. No tópico "Avaliação de risco", são apresentados o objetivo, os itens para avaliação e a escala de risco ELPO.

No item "Superfícies e dispositivos", recomendam-se: verificar os dispositivos disponíveis na instituição e utilizá-los conforme o peso e a capacidade necessários para a movimentação segura do paciente; posicionar o paciente em superfície lisa e que redistribua a pressão; e usar curativos profiláticos para proeminências ósseas e outras áreas sujeitas à pressão, à fricção ou a corte. Ainda, tem-se o alerta para não posicionar o paciente em colchão térmico sem proteção e não utilizar toalhas, lençóis ou cobertores como dispositivo de posicionamento.

No tópico "Práticas gerais de posicionamento", são indicados: manter o alinhamento corporal do paciente; ter número de pessoas suficiente durante a movimentação do paciente; proteger o corpo do paciente de superfícies metálicas e áreas de pressão; proceder com proteção ocular; evitar hiperextensão cervical; usar faixa de segurança; e monitorar o posicionamento durante o intraoperatório.

Para cada posição cirúrgica, um desenho representativo ao posicionamento antecede a descrição, por tópicos, de como proceder. Após cada descrição de posição cirúrgica, foi inserida uma caixa de lembrete de atenção para o posicionamento, e, na sequência, foram apontados os riscos envolvidos.

As posições cirúrgicas com seus devidos riscos estão no Quadro 1.

Construído o manual, o arquivo foi enviado para avaliação das enfermeiras perioperatórias (gerente e coordenadora) do BC, com a apresentação da proposta do conteúdo, material e leiaute. Elas não viram necessidade de alterações, validando o material e encaminhando-o à impressão colorida e encadernação. Duas cópias foram apresentadas à equipe de enfermagem como guia norteador para posicionamento e estratégias para prevenção de lesões de pele.

Foi solicitada à equipe a leitura do material durante o horário de trabalho, conforme suas atividades no plantão, tarefa que levou cerca de três dias para abranger a equipe em vários turnos. Ao término do processo, o livreto ficou disponível para consulta no posto de enfermagem.

O manual, na forma de arquivo, também foi enviado para o setor de qualidade da instituição, como produto do plano de ação, como medida de prevenção de lesões por posicionamento cirúrgico.

\section{DISCUSSÃO}

Não há descrição na literatura sobre a construção de manuais para posicionamento cirúrgico. Em geral, aprende-se acerca do tema durante a formação profissional, e algumas publicações apresentam um direcionamento a respeito das posições cirúrgicas e os cuidados assistenciais.

O enfermeiro é o responsável pelo planejamento e pela implementação de intervenções que previnam as complicações decorrentes do procedimento anestésico-cirúrgico, prestando assistência ao paciente juntamente com a equipe multiprofissional e decidindo, assim, o melhor posicionamento 
Quadro 1. Posições cirúrgicas, posicionamento e riscos descritos no tópico “Posições cirúrgicas” do manual de posicionamento cirúrgico.

Posição

cirúrgica

Posicionamento

Riscos envolvidos

Posicione os membros superiores do paciente, conforme a necessidade da equipe cirúrgica ou as limitações do paciente. Nos casos em que os membros superiores são ao longo do corpo: coloque os membros superiores em posição neutra, com as palmas da mão voltadas para o corpo; não hiperextenda os cotovelos; prenda o tecido que envolve os membros superiores entre o corpo e braço do paciente; assegure-se de que o tecido que prende o membro superior do paciente esteja apertado o suficiente para proteger o membro superior, mas não tão apertado para criar um ponto de pressão. Em casos com membros superiores em braçadeira: coloque a

Supina braçadeira na altura da mesa cirúrgica; mantenha os membros superiores em ângulo inferior a 90; posicione os membros superiores com a palma para cima; mantenha o alimento dos membros superiores.

Flexione os joelhos do paciente em aproximadamente de 5 a $10^{\circ}$. Coloque a faixa de segurança aproximadamente

$5 \mathrm{~cm}$ acima dos joelhos do paciente.

Eleve os calcanhares do paciente para fora da superfície subjacente. Redistribua a pressão dos calcanhares

com dispositivos de posicionamento.

É recomendado o uso de dispositivos adesivos para áreas de pressão em procedimentos com longa duração.

Posicione a cabeça do paciente em uma posição neutra, sem flexão excessiva, extensão ou rotação.

Use um posicionador facial quando a cabeça do

paciente estiver posicionada na linha média.

Monitore a posição do rosto do paciente.

Evite a pressão direta sobre os olhos do paciente.

Posicione os membros superiores conforme determinado pelas necessidades da equipe cirúrgica e as limitações físicas do paciente. Quando os membros superiores são ao longo do corpo: coloque os membros superiores em posição neutra, com as palmas da mão voltadas para o corpo; não hiperextenda os cotovelos; prenda o tecido que envolve os membros superiores entre o corpo e o braço do paciente; assegure-se de que o tecido que prende o membro superior do paciente esteja apertado o suficiente para proteger o membro superior, mas não tão apertado para criar um ponto de pressão. Com membros superiores em braçadeira: coloque a braçadeira no

Prona nível inferior ao tórax abduzindo os membros superiores em menos de $90^{\circ}$ com cotovelos flexionados, palmas voltadas para baixo; mantenha os membros superiores em alinhamento neutro; não posicione os membros superiores acima da cabeça.

Posicione o tórax do paciente sobre dois rolos posicionadores da clavícula até a crista ilíaca.

Posicione as mamas, o abdômen e as genitais de maneira que estejam livres de pressão ou torção.

Coloque um rolo posicionador na crista ilíaca.

Proteja os joelhos do paciente.

Eleve os pés do paciente e coloque protetores sob as pernas, para deixar os pés mais altos e livres.

Avalie os pulsos do paciente após o posicionamento

e o alinhamento corporal.

Posicione o paciente em de 5 a $10^{\circ} \mathrm{em}$ Trendelenburg.

É recomendado o uso de dispositivos adesivos em áreas de pressão (mento, clavícula, tórax, ilíaca, joelhos).
Lombalgia, por conta da perda da curvatura lombar normal.

Lesão de nervo ulnar e radial.

Lesão de plexo braquial.

Lesão de plexo cervical ou lesão medular por hiperextensão da cabeça.

Alopecia, por compressão

dos folículos pilosos.

Lesão isquêmica por pressão.

Lesão cervical, pela rotação do pescoço.

Edema ocular ou cegueira.

Compressão ou lesões isquêmicas das estruturas faciais.

Compressão abdominal com diminuição do retorno venoso.

Lesão de plexo braquial.

Lesão de nervo ulnar e nervo radial.

Compressão de genitália em homens, causando edema, hematoma e isquemia. Compressão de mamas nas mulheres. 
Quadro 1. Continuação.

\begin{tabular}{|c|c|c|}
\hline $\begin{array}{l}\text { Posição } \\
\text { cirúrgica }\end{array}$ & Posicionamento & Riscos envolvidos \\
\hline Lateral & $\begin{array}{l}\text { Coloque um posicionador de cabeça ou travesseiro sob a cabeça do paciente. } \\
\text { Avalie e monitore a orelha dependente do paciente após posicionamento. } \\
\text { Apoie e proteja os membros superiores em dois níveis e com } \\
\text { braçadeiras paralelas, com um membro em cada braçadeira e ambos } \\
\text { com abertura inferior a } 90^{\circ} \text {. } \\
\text { Coloque um rolo posicionador sob o tórax dependente do paciente, no } \\
\text { nível da sétima à nona costela. } \\
\text { Verifique os pulsos radiais bilaterais do paciente } \\
\text { após a colocação do rolo axilar. } \\
\text { Mantenha o alinhamento espinhal fisiológico do paciente. } \\
\text { Coloque a faixa de segurança no quadril do paciente. } \\
\text { Flexione o membro inferior dependente do paciente no quadril e no joelho. } \\
\text { Posicione o membro inferior superior do paciente e apoie com } \\
\text { travesseiro entre as pernas. } \\
\text { Minimize o grau de flexão da mesa cirúrgica e a elevação lateral na } \\
\text { altura do rim o máximo possível. } \\
\text { Coloque a faixa de segurança na crista ilíaca para procedimentos torácicos } \\
\text { e ortopédicos. Para procedimentos renais, acrescente uma faixa no tórax. }\end{array}$ & $\begin{array}{l}\text { Lesão cervical por flexão, extensão ou } \\
\text { rotação exagerada do pescoço. } \\
\text { Lesão de plexo braquial, apresentando-se } \\
\text { como paresia, dor, parestesia e diminuição } \\
\text { da força em membros superiores. } \\
\text { Abrasões corneanas, edema ocular, perda } \\
\text { visual parcial e total. } \\
\text { Necrose isquêmica em orelha. } \\
\text { Lesão do nervo fibular, por compressão pelo } \\
\text { peso superior do joelho. } \\
\text { Necrose de fêmur, por compressão da } \\
\text { cabeça do fêmur no acetábulo pela fita de } \\
\text { fixação lateral ou por compressão da artéria } \\
\text { femoral pelos posicionadores. }\end{array}$ \\
\hline Trendelenburg & $\begin{array}{l}\text { Coloque os membros superiores ao lado do corpo. } \\
\text { Minimize o grau de Trendelenburg o máximo possível. } \\
\text { Implemente medidas para evitar que o paciente deslize na mesa cirúrgica. } \\
\text { Evite o uso de ombreiras, se possível. } \\
\text { Evite essa posição para pacientes que são obesos mórbidos extremos. }\end{array}$ & $\begin{array}{l}\text { Lesão de nervo ulnar. } \\
\text { Lesão isquêmica por pressão. } \\
\text { Aumento da pressão intracraniana. } \\
\text { Alterações respiratórias, provocadas pela } \\
\text { compressão das bases pulmonares pelas } \\
\text { vísceras abdominais. }\end{array}$ \\
\hline $\begin{array}{l}\text { Trendelenburg } \\
\text { reverso }\end{array}$ & $\begin{array}{l}\text { Utilize uma peseira para evitar que o paciente deslize e se reduza o } \\
\text { potencial de lesão dos nervos e flexão do tornozelo. } \\
\text { Monitorar os pés do paciente e implementar } \\
\text { ações corretivas são indicados. }\end{array}$ & $\begin{array}{l}\text { Lesão de plexo braquial, nervo ulnar. } \\
\text { Lesão isquêmica por pressão. } \\
\text { Deslizamento do paciente ou até mesmo } \\
\text { queda da mesa cirúrgica. } \\
\text { Embolia venosa. }\end{array}$ \\
\hline Litotomia & $\begin{array}{l}\text { Posicione a nádega do paciente na parte final da mesa cirúrgica, de } \\
\text { maneira que o corpo suporte a região sacra na superfície da mesa. } \\
\text { Proteja as mãos e os dedos do paciente de lesões quando as } \\
\text { perneiras são colocadas ou quando são } \\
\text { movimentados os membros inferiores. } \\
\text { Posicione os quadris do paciente de forma a evitar } \\
\text { flexão excessiva, rotação ou abdução. } \\
\text { Coloque as perneiras em altura uniforme. } \\
\text { Lenta e simultaneamente, coloque os membros } \\
\text { inferiores do paciente nas perneiras. } \\
\text { Apoie o membro inferior do paciente } \\
\text { sobre a maior superfície possível. } \\
\text { Coloque os calcanhares do paciente na parte mais baixa possível. } \\
\text { Não permita que os membros inferiores do } \\
\text { paciente sejam apoio para a perneira. } \\
\text { Ao final do procedimento, retire os membros inferiores da perneira } \\
\text { lentamente e flexione-os antes de apoiá-los na mesa cirúrgica. }\end{array}$ & $\begin{array}{l}\text { Parestesia na distribuição nervosa afetada } \\
\text { é a complicação mais comum, podendo } \\
\text { ocorrer lesão do nervo obturador, do nervo } \\
\text { cutâneo femoral lateral, do nervo ciático, do } \\
\text { nervo fibular e do nervo femoral. } \\
\text { Trombose venosa profunda. } \\
\text { Síndrome do compartimento das } \\
\text { extremidades inferiores. }\end{array}$ \\
\hline $\begin{array}{l}\text { Fowler ou } \\
\text { sentada }\end{array}$ & $\begin{array}{l}\text { Mantenha a cabeça do paciente em posição neutra, sem flexão } \\
\text { excessiva, extensão ou rotação. } \\
\text { Flexione e proteja os membros superiores do paciente ou membro } \\
\text { não operado junto ao corpo. } \\
\text { Apoie a região sacra do paciente. } \\
\text { Flexione os joelhos do paciente em } 30^{\circ} \text {. } \\
\text { Impeça que o abdômen do paciente } \\
\text { descanse sobre os membros inferiores. } \\
\text { Coloque a faixa de segurança sobre as coxas do paciente. }\end{array}$ & $\begin{array}{l}\text { Flexão cervical excessiva, podendo impedir o } \\
\text { fluxo sanguíneo arterial e venoso, causando } \\
\text { hipoperfusão cerebral ou congestão venosa } \\
\text { do cérebro, além de torção do tubo e edema } \\
\text { na face e na língua. } \\
\text { Lesões de plexo braquial, } \\
\text { nervo ulnar e ciático. } \\
\text { Cegueira. } \\
\text { Embolia gasosa. }\end{array}$ \\
\hline
\end{tabular}


para o paciente, de modo a facilitar as atividades durante o ato anestésico-cirúrgico?.

Qualquer paciente submetido a um procedimento cirúrgico possui risco para lesão decorrente de posicionamento. Essas lesões podem ser causadas por alongamento ou compressão dos tecidos, que levam à isquemia, por friç̧ão e cisalhamento, ou por pressão prolongada ${ }^{2}$.

Em um estudo realizado em Minas Gerais, a incidência de lesões decorrentes do posicionamento cirúrgico foi de $74 \%$, caracterizadas como de grau I, com localização mais frequente na região sacral, seguida da calcânea ${ }^{10}$. Em São Paulo, um estudo somente com cirurgias cardíacas obteve $19 \%$ de lesões por posicionamento, também em sua maioria de grau $\mathrm{I}^{11}$.

Prevenir lesões por posicionamento é responsabilidade da equipe cirúrgica, envolvendo enfermagem, anestesista, cirurgião e assistente. Faz-se necessária atenção às condições de suporte de apoio, ao tempo de uso durante o procedimento e a qualquer condição que possa interferir no posicionamento do paciente ${ }^{2,12}$.

Compreender as áreas de pressão para cada posição cirúrgica e as intervenções básicas para prevenção de LP, por vezes, não é domínio da equipe de enfermagem, o que torna o manual de posicionamento um direcionador para assistência.

As intervenções eficazes na prevenção de lesões de pele estão relacionadas ao alívio de pressões durante e imediatamente após a permanência do paciente na mesa cirúrgica, sobre o colchão padrão ${ }^{12}$.

O uso de imagens ilustrativas para cada posição cirúrgica e os dispositivos disponíveis na instituição auxiliam a equipe de enfermagem a compreender melhor como proceder e quais recursos podem ser aplicados.

Alguns técnicos de enfermagem relataram, durante a leitura do material, que desconheciam alguns dos tópicos elucidados e que iriam ficar mais atentos às recomendações durante suas atividades diárias.

Outra característica é o número insuficiente de enfermeiros assistenciais no CC, o que impossibilita a presença desses profissionais na realização de todos os posicionamentos. Dessa forma, essa atividade fica a critério do técnico de enfermagem (circulante de SO) e da equipe médica.

Assim, durante a capacitação de sua equipe, o enfermeiro perioperatório tem a oportunidade de desenvolver ações educativas para garantir a segurança do paciente cirúrgico e a redução dos riscos de lesões por posicionamento cirúrgico ${ }^{5}$.

Não foram encontrados estudos para verificar o conhecimento dos profissionais integrantes da equipe de enfermagem sobre as áreas de pressão ou como proceder na realização dos diferentes posicionamentos cirúrgicos, porém entende-se que pode haver falhas nesses processos por falta de conhecimento.

Atualmente, os autores de um estudo sobre posicionamento para cirurgias robóticas urológicas descreveram o protocolo de posicionamento para esse perfil de pacientes, envolvendo o uso de dispositivos recomendados para prevenção ${ }^{5}$, entretanto mais estudos com relação de risco e recomendação devem ser desenvolvidos.

Não houve resistência dos profissionais de enfermagem quanto ao uso do manual de posicionamento, e a leitura do conteúdo foi interpretada como aquisição de conhecimento profissional.

Ainda se buscam a sistematização e o maior emprego de recursos para melhoria do processo, mas um conteúdo direcionador é um primeiro passo para a conscientização dos profissionais atuantes na enfermagem perioperatória.

Espera-se que esta pesquisa contribua com o conhecimento dos profissionais de enfermagem para promover o adequado posicionamento cirúrgico e prevenir a ocorrência de possíveis danos decorrentes de EA.

Entre as limitações deste estudo, pode-se considerar a adequação dos recursos disponíveis à demanda do serviço da instituição, de modo a abranger um amplo número de profissionais de enfermagem com diferentes níveis de conhecimento. Sistematizar o processo do posicionamento cirúrgico no que tange ao escore de risco ainda é um desafio a ser superado.

\section{CONSIDERAÇÕES FINAIS}

O manual de posicionamento cirúrgico desenvolvido em uma instituição hospitalar de alta complexidade de São Paulo, baseado na literatura vigente, contempla os riscos, as diferentes posições cirúrgicas e as superfícies de suporte disponíveis, visando direcionar os profissionais de enfermagem para a provisão de um adequado posicionamento cirúrgico, com uso de recursos de proteção que previnam a ocorrência de lesões. As ilustrações permitem elucidar a posição e seus cuidados, e a disposição de cores chama a atenção do leitor.

Após leitura e o conhecimento da equipe do manual norteador, este ficou disponível para consulta por qualquer membro da equipe no posto de enfermagem do CC, podendo ser acessado a qualquer momento.

Com uma equipe de enfermagem que domine as diferentes posições cirúrgicas e atuantes na prevenção de LP, os índices de EA relacionados às LP tendem a reduzir-se. 


\section{REFERÊNCIAS}

1. Lopes CMM, Galvão CM. Posicionamento cirúrgico: evidências para o cuidado de enfermagem. Rev Latino-Am Enfermagem. 2010;18(2):28794. http://dx.doi.org/10.1590/S0104-11692010000200021

2. Burglingame BL. Guideline implementation: positioning the patient. AORN J. 2017;106(3):227-37. https://doi.org/10.1016/j. aorn.2017.07.010

3. Spruce L. Back to Basics: preventing perioperative pressure injuries. AORN J. 2017;105(1):92-9. https://doi.org/10.1016/j.aorn.2016.10.018

4. Miranda AB, Fogaça AR, Rizzetto M, Lopes LCC. Posicionamento cirúrgico: cuidados de enfermagem no transoperatório. Rev SOBECC. 2016;21(1):52-8. https://doi.org/10.5327/Z1414-4425201600010008

5. Angelo CS, Pachioni CFM, Joaquim EHG, Silva EAL, Santos GG, Bonfim IM, et al. Efetividade do protocolo prevenção de lesões de pele em cirurgias urológicas robóticas. Rev SOBECC. 2017;22(3):15260. https://doi.org/10.5327/Z1414-4425201700030006

6. Munro $\mathrm{C}$. The development of a pressure ulcer risk-assessment scale for perioperative patients. AORN J. 2010;92(3):272-87. https://doi. org/10.1016/j.aorn.2009.09.035
7. Lopes CMM, Haas VJ, Dantas RAS, Oliveira CG, Galvão CM. Escala de avaliação de risco para lesões decorrentes do posicionamento cirúrgico. Rev Latino-am. Enfermagem. 2016;24:e2704. https://doi. org/10.1590/1518-8345.0644.2704

8. Anderson O, Davis R, Hanna GB, Vicent CA. Surgical adverse events: a systematic review. Am J Surg. 2013;206(2):253-62. https://doi. org/10.1016/j.amjsurg.2012.11.009

9. Barbosa MH, Oliva AMB, Sousa Neto AL. Ocorrência de lesões perioperatórias por posicionamento cirúrgico. Rev Cuba Enferm [Internet]. 2011 [citado 2017 Nov 10];27(1):31-41. Disponível em: http://www.medigraphic.com/pdfs/revcubenf/cnf-2011/cnf111e.pdf

10. Carneiro GA, Leite RCBO. Lesões de pele no intraoperatório de cirurgia cardíaca: incidência e caracterização. Rev Esc Enferm USP. 2011;45(3):611-6. http://dx.doi.org/10.1590/ S0080-62342011000300009

11. Grigoleto ARL, Avelar MCQ, Lacerda RA, Mendonça SHF. Complicações decorrentes do posicionamento cirúrgico de clientes idosos submetidos à cirurgia de quadril. Esc Anna Nery. 2011;15(3):531-5. http://dx.doi. org/10.1590/S1414-81452011000300013 